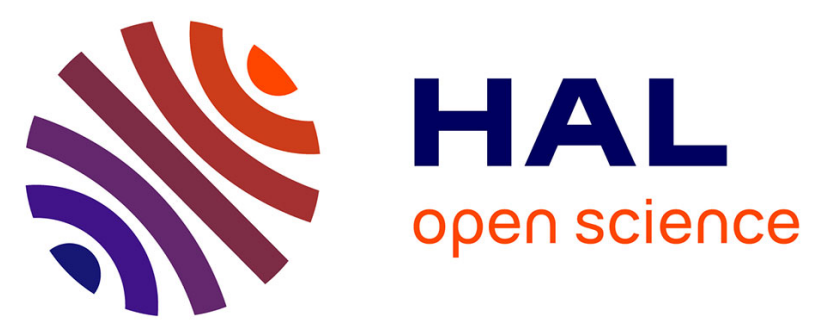

\title{
Lur'e Postnikov Lyapunov functional technique to global Mittag-Leffler stability of fractional-order neural networks with piecewise constant argument
}

\author{
Li-Fei Wang, Huaiqin Wu, Da-Yan Liu, Driss Boutat, Yi-Ming Chen
}

\section{To cite this version:}

Li-Fei Wang, Huaiqin Wu, Da-Yan Liu, Driss Boutat, Yi-Ming Chen. Lur'e Postnikov Lyapunov functional technique to global Mittag-Leffler stability of fractional-order neural networks with piecewise constant argument. Neurocomputing, 2018, 302, pp.23-32. 10.1016/j.neucom.2018.03.050 . hal02884224

\author{
HAL Id: hal-02884224 \\ https://hal.science/hal-02884224
}

Submitted on 29 Jun 2020

HAL is a multi-disciplinary open access archive for the deposit and dissemination of scientific research documents, whether they are published or not. The documents may come from teaching and research institutions in France or abroad, or from public or private research centers.
L'archive ouverte pluridisciplinaire HAL, est destinée au dépôt et à la diffusion de documents scientifiques de niveau recherche, publiés ou non, émanant des établissements d'enseignement et de recherche français ou étrangers, des laboratoires publics ou privés. 


\title{
Lur'e Postnikov Lyapunov functional technique to global Mittag-Leffler stability of fractional-order neural networks with piecewise constant argument
}

\author{
Li-Fei Wang ${ }^{\mathrm{a}}$, Huaiqin Wu ${ }^{\mathrm{a}, *}$, Da-Yan Liu ${ }^{\mathrm{b}}$, Driss Boutat ${ }^{\mathrm{b}}$, Yi-Ming Chen ${ }^{\mathrm{a}, \mathrm{c}, \mathrm{d}}$ \\ ${ }^{a}$ School of Science, Yanshan University, Qinhuangdao 066001, China \\ ${ }^{b}$ INSA Centre Val de Loire, Université d'Orléans, PRISME EA 4229, Bourges Cedex 18020, France \\ ${ }^{c}$ Le STUDIUM RESEARCH PROFESSOR, Loire Valley Institute for Advanced Studies, Orléans, France \\ ${ }^{d}$ PRISME (INSA-Institut National des Sciences Appliquées)-88, Boulvevard Lahitolle, 18000 Bourges, France
}

\begin{abstract}
In this paper, the global Mittag-Leffler stability issue of fractional-order neural networks (FNNs) with piecewise constant argument is investigated. Firstly, a new inequality with respect to the fractional derivative of integer-order variable upper limit integral is proposed, which not only is favorable to the construction of Lyapunov function but also enriches the fractional order calculus theory. Secondly, based on topological degree theory, the existence and uniqueness of equilibrium point is certified. In addition, under some suitable assumptions, by applying Picard successive approximation technique, the proof of the existence and uniqueness of solution with the initial value is given. Moreover, by introducing integral term into Lyapunov functional, Lur'e Postnikov type Lyapunov functional is constructed, and a sufficient condition is addressed in terms of linear matrix inequalities (LMIs) to guarantee that the considered FNNs are Mittag-Leffler stable. Finally, an example is given to demonstrate the validity of the obtained results.
\end{abstract}

Keywords: Fractional neural network; Mittag-Leffler stability; Piecewise constant argument; Lur'e Postnikov type Lyapunov function; LMIs;

\section{Introduction}

Since Mcculloch and Pitts firstly put forward the mathematical model of neural networks on the basis of the characteristic analysis of the neurons [1], neural networks have become the hottest issues and have been applied widely in many fields, such as the artificial signal and image processing, switching in electronic circuits, automatic control and so on. It deserves to point that, in 1982 [2], Hopfield proposed a famous Hopfield neural networks model, and analyzed the stability of networks by utilizing energy function approach firstly, which set off a wave of worldwide researches on neural networks, such as the research on dynamic characteristics of neural networks [3].

Compared with integer-order calculus, fractionalorder calculus provides a more accurate instrument for the description of memory and hereditary properties of various processes. Nowadays, it has found that fractional calculus can be applied in viscoelastic system,

\footnotetext{
*Corresponding author

Email address: huaiqinwu@ysu. edu.cn (Huaiqin Wu)
}

electric polarization, electromagnetic waves, colored noise, heat conduction, robotics, biological system, finance and so on [4-11], which can not be done by integer order calculus.

It is well known that neural networks can be utilized to deal with many computational problems, which requires the networks to accomplish some dynamical behaviors such as the stability. Only when neural networks is stable, can it operates normally. So, the studying stability of neural networks is essential. Recently, some excellent results in the stability analysis of FNNs have been derived. In [12], Wu et al discussed Quasi-uniform stability for FNNs with mixed time-varying delays. In [13-15], the researchers discussed different FNNs and established some sufficient conditions for global MittagLeffler stability and synchronization. In [16], Zhang et al addressed the stability analysis for fractional-order Hopfield neural networks with discontinuous activation functions. In [17-18], Rakkiyappan et al discussed the stability and asymptotical periodicity of a fractionalorder complex-valued neural network with constant delays and time varying delays. 
The dynamic study of differential equations with piecewise constant argument was initiated by Cooke and Wiener in [22]. Subsequently, an increasing number of scholars are inspired to investigate differential equations with piecewise constant argument, owing to the fact that it represents a hybrid of continuous and discrete dynamical systems, and combines with the properties of both the differential and difference equations. Based on the above reason, differential equations with piecewise constant argument can play a crucial role in handling many real world problems in [23-26], such as oscillatory motion, mechanical, biological system and so on. Generally, the differential equation with piecewise constant argument is investigated by reducing the equation to an equivalent discrete equation [22,27]. However, this method has heavy limitations to non-integer piecewise constant argument. Recently, in [28,29], Akhmet reduced the differential equation with piecewise constant argument to an equivalent integral equation, which greatly promotes the application of this equation. It is worthy to mention that the arguments in the differential equation with piecewise constant argument are not only delayed but advanced. In [30, 31], Akhmet discussed the global stability of recurrent neural networks and cellular neural networks with piecewise constant argument, respectively. In [32], Yu et al studied the stability of neural networks with periodic coefficients and piecewise constant argument. In [33], the robust stability of interval fuzzy Cohen-Grossberg neural networks with piecewise constant argument of generalized type was investigated. The synchronization issue of complex dynamical networks with piecewise constant argument of generalized type was considered in [34]. Very recently, in [35], Wu et al studied the Mittag-Leffler stability of FNNs in the presence of generalized piecewise constant arguments, the sufficient condition is given to guarantee that the considered FNNs is Mittag-Leffler stable.

It should be pointed out that, in the aforementioned literature with respect to the stability of FNNs, the Lyapunov function for solving the stability issue is the absolute value function $V(t)=\sum_{i=0}^{n}\left|x_{i}\right|$, see [13-19], or the positive definite quadratic function $V(t)=x^{T} P x$, see [20-21, 35]. It is easy to see, the information from neuron activation functions of the network is not applied in the Lyapunov function construction. Hence, the obtained stability results in the above papers have a certain degree of conservatism. However, the introduction of Lur'e Postnikov type Lyapunov functional could overcome this shortcoming.

Motivated by the above discussions, in this paper, our aim is to investigate the gtlobal Mittag-Leffler stability of FNNs with piecewise constant argument of generalized type by applying Lur'e Postnikov type Lyapunov functional approach. The crucial novelties of our contribution lie in following aspects: 1. A new inequality with respect to the fractional derivative of integer-order variable upper limit integral is developed; 2 . The existence and uniqueness of equilibrium point and solution for FNNs with piecewise constant argument are certified, respectively; 3. The integral term $\int_{0}^{z_{i}(t)} f_{i}(s) d s$ is introduced into Lur'e Postnikov Lyapunov functional, and the Mittag-Leffler stability condition is presented in terms of LMIs.

The rest of this paper is organized as follows. In Section 2, some definitions and lemmas are given, and model description is introduced. In Section 3, the existence and uniqueness of equilibrium point and solution with initial value are proved, respectively. Some sufficient criteria for the global Mittag-Leffler stability are derived. In Section 4, a numerical example is offered to verify the validity of the obtained results. The conclusion is drawn in Section 5.

Notation: $R$ denotes the set of real numbers, $N$ and $R^{+}$ represent the sets of natural and nonnegative real numbers, respectively. $R^{n}$ denotes the $n$-dimensional Euclidean space, $R^{m \times n}$ denotes the set of all $m \times n$ real matrices. Given column vectors $x=\left(x_{1}, x_{2}, \cdots, x_{n}\right)^{T} \in R^{n}$ and $y=\left(y_{1}, y_{2}, \cdots, y_{n}\right)^{T} \in R^{n}, x^{T} y=\sum_{i=1}^{n} x_{i} y_{i}$ denotes the scalar product of $x$ and $y$, where the superscript $T$ represents the transpose operator. $\|x\|=\left(\sum_{i=1}^{n} x_{i}^{2}\right)^{\frac{1}{2}}$ denotes the vector norm of $x$. For real symmetric $P, P>0$ $(P<0)$ means that the matrix $P$ is a real symmetric positive (negative) definite matrix, $\lambda_{\max }(P)$ and $\lambda_{\min }(P)$ stand for the maximal and minimal eigenvalues of $P$, respectively. We take two real-valued sequence $\theta_{i}, \xi_{i}$, $i \in N$ such that $0<\theta_{i}<\theta_{i+1}, \theta_{i}<\xi_{i}<\theta_{i+1}$ for all $i \in N, \theta_{i} \rightarrow+\infty$.

\section{Preliminaries and model description}

\subsection{Caputo fractional-order derivative}

In this subsection, we recall some basic definitions and lemmas with respect to fractional calculus.

Definition 2.1. ([36]) The fractional integral of order $\alpha$ for an integrable function $f(t):[0,+\infty) \rightarrow R$ is defined as

$$
{ }_{0}^{R} I_{t}^{\alpha} f(t)=\frac{1}{\Gamma(\alpha)} \int_{0}^{t} \frac{f(\tau)}{(t-\tau)^{1-\alpha}} d \tau,
$$

where $0<\alpha<1$, and $\Gamma(\cdot)$ is the Gamma function which is defined by $\Gamma(z)=\int_{0}^{\infty} e^{-t} t^{z-1} d t(\operatorname{Re}(z)>0)$, where $\operatorname{Re}(z)$ is the real part of $z$. 
Definition 2.2. ([36]) Caputo's fractional derivative of order $\alpha$ for a function $f \in C^{n}([0,+\infty], R)$ is defined by

$$
{ }_{0}^{C} D_{t}^{\alpha} f(t)=\frac{1}{\Gamma(n-\alpha)} \int_{0}^{t} \frac{f^{n}(\tau)}{(t-\tau)^{\alpha-n+1}} d \tau,
$$

where $t \geqslant 0$ and $n$ is a positive integer such that $n-1<$ $\alpha<n \in N$. Particularly, when $0<\alpha<1$,

$$
{ }_{0}^{C} D_{t}^{\alpha} f(t)=\frac{1}{\Gamma(1-\alpha)} \int_{0}^{t} \frac{f^{\prime}(\tau)}{(t-\tau)^{\alpha}} d \tau .
$$

Caputo's fractional derivative ${ }_{0}^{C} D_{t}^{\alpha}$ has the following properties:

Lemma 2.1. ([37]).

(i) ${ }_{0}^{C} D_{t}^{\alpha}{ }_{0}^{R} I_{t}^{\beta} x(t)={ }_{0}^{C} D_{t}^{\alpha-\beta} x(t)$, where $\alpha \geq \beta \geq 0$. Especially, when $\alpha=\beta,{ }_{0}^{C} D_{t}^{\alpha}{ }_{0}^{R} I_{t}^{\beta} x(t)=x(t)$.

(ii) Let $n=[\alpha]+1$ for $\alpha \notin N$ or $n=\alpha$ for $\alpha \in N$. If $x(t) \in C^{n}[0,+\infty)$, then

$$
{ }_{0}^{R} I_{t}^{\alpha}{ }_{0}^{C} D_{t}^{\alpha} x(t)=x(t)-\sum_{k=0}^{n-1} \frac{x^{(k)}(0)}{k !} t^{k}, \quad t>0 .
$$

In particular, if $0<\alpha \leq 1$ and $x(t) \in C^{1}[0,+\infty]$, then ${ }_{t}^{R} I_{0}^{\alpha}{ }_{t}^{C} D_{0}^{\alpha} x(t)=x(t)-x(0)$.

(iii) Let $c$ be any constant, then ${ }_{0}^{C} D_{t}^{\alpha} c=0$.

(iv) ${ }_{0}^{C} D_{t}^{\alpha}\left(v_{1} f(t)+v_{2} g(t)\right)=v_{1}{ }_{0}^{C} D_{t}^{\alpha} f(t)+v_{2}{ }_{0}^{C} D_{t}^{\alpha} g(t)$, where $v_{1}$ and $v_{2}$ are any constants.

Similar to the exponential function frequently used in the solutions of integer-order systems, a function frequently used in the solution of fractional-order systems is the Mittag-Leffler function.

Definition 2.3. ([37]). (Mittag-Leffler function) A twoparameter Mittag-Leffler function is defined as

$$
E_{\alpha, \beta}(z)=\sum_{k=0}^{\infty} \frac{z^{k}}{\Gamma(\alpha k+\beta)},
$$

where $\alpha>0, \beta>0$ and $z \in C$. For $\beta=1$, its oneparameter form is shown as

$$
E_{\alpha}(z)=\sum_{k=0}^{\infty} \frac{z^{k}}{\Gamma(\alpha k+1)}=E_{\alpha, 1}(z),
$$

especially $E_{1,1}(z)=e^{z}$, where $\alpha=1, \beta=1$.

Lemma 2.2. ([13]) If $V(t)$ is a continuous function on $[0,+\infty)$ and satisfies

$$
{ }_{0}^{C} D_{t}^{\alpha} V(t) \leq \theta V(t),
$$

where $0<\alpha<1$ and $\theta$ is a constant, then

$$
V(t) \leq V(0) E_{\alpha}\left(\theta t^{\alpha}\right), \quad t \geq 0 .
$$

Lemma 2.3. Given any scalar $\epsilon>0, x, y \in R^{n}$ and matrix $A$, then

$$
x^{T} A y \leq \frac{1}{2 \epsilon} x^{T} A A^{T} x+\frac{\epsilon}{2} y^{T} y
$$

\subsection{Model description}

In this subsection, we give the network model description and propose some rational assumptions which are needed in the development of this paper.

Consider the following FNNs described by differential equations with piecewise constant argument of generalized type:

$$
{ }_{0}^{C} D_{t}^{\alpha} x(t)=-A x(t)+B f(x(t))+C g(x(\gamma(t)))+I,
$$

or equivalently

$$
\begin{aligned}
{ }_{0}^{C} D_{t}^{\alpha} x_{i}(t)= & -a_{i} x_{i}(t)+\sum_{j=1}^{n} b_{i j} f_{j}\left(x_{j}(t)\right)+\sum_{j=1}^{n} c_{i j} g_{j}\left(x_{j}(\gamma(t))\right) \\
& +I_{i}, \quad i=1,2, \cdots, n,
\end{aligned}
$$

where $0<\alpha<1$. If $t \in\left[\theta_{k}, \theta_{k+1}\right), k \in N, t \in R^{+}$, then $\gamma(t)=\zeta_{k} . n$ corresponds to the number of units in a neural network. $x(t)=\left(x_{1}(t), \cdots, x_{n}(t)\right)^{T} \in R^{n}$, $x_{i}(t)$ stands for the state of the ith unit at time $t$. $A=$ $\operatorname{diag}\left(a_{1}, a_{2}, \cdots, a_{n}\right)$ represents self connection weight, where $a_{i}>0 . B=\left(b_{i j}\right)_{n \times n}$ represents interconnection weight matrix, where $b_{i j}$ denotes the strength of the $j t h$ unit on the ith unit at time $t . \quad C=\left(c_{i j}\right)_{n \times n}$ also represents interconnection weight matrix, where $c_{i j}$ infers the strength of the $j t h$ unit on the ith unit at time $\gamma(t)$. $f, g: R^{n} \rightarrow R^{n}$ are diagonal mapping, $g(x(\gamma(t)))=$ $\left(g_{1}\left(x_{1}(\gamma(t))\right), g_{2}\left(x_{2}(\gamma(t))\right), \cdots, g_{n}\left(x_{n}(\gamma(t))\right)\right)^{T}, f(x(t))=$ $\left(f_{1}\left(x_{1}(t)\right), f_{2}\left(x_{2}(t)\right), \cdots, f_{n}\left(x_{n}(t)\right)\right)^{T}$, where $f_{j}$ and $g_{j}$ denote the measures of activation to its incoming potential of the units $j$ at time $\gamma(t)$ and $t$, respectively. $I=$ $\left(I_{1}, I_{2}, \cdots, I_{n}\right)^{T}$ is the external input vector.

The network system (1) is of mixed type system, that is, the argument $\gamma(t)$ can change its deviation character during the motion. Fix $k \in N$, and consider system (1) on the interval $\left[\theta_{k}, \theta_{k+1}\right)$, the identification function $\gamma(t)$ is equal to $\zeta_{k}$. If $t$ satisfies $\theta_{k} \leq t<\zeta_{k}$, then $\gamma(t)>t$, and system (1) is an equation with advanced argument. Similarly, if $\zeta_{k} \leq t<\theta_{k+1}$, then $\gamma(t)<t$, and system (1) is an equation with delayed argument. Consequently, system (1) changes the type of deviation of the argument during the process.

The following assumptions will be needed throughout the paper:

(H1) the activation functions $f_{j}, g_{j}$ satisfy $f_{j}(0)=0$, $g_{j}(0)=0$ for each $j=1,2, \cdots n$. 
(H2) there exists constants $L_{i}^{1}, L_{i}^{2}, L_{i}^{3}, L_{i}^{4}>0$ such that

$$
L_{i}^{1}<\frac{f_{i}(u)-f_{i}(v)}{u-v}<L_{i}^{2}, L_{i}^{3}<\frac{g_{i}(u)-g_{i}(v)}{u-v}<L_{i}^{4}
$$

for all $u, v \in R^{n}, i=1,2, \cdots, n . L^{2}=\operatorname{diag}\left(L_{1}^{2}\right.$,

$$
\left.L_{2}^{2}, \cdots, L_{n}^{2}\right), L^{4}=\operatorname{diag}\left(L_{1}^{4}, L_{2}^{4}, \cdots, L_{n}^{4}\right) \text {. }
$$

(H3) there exists a positive number $\theta$ such that $\theta_{i+1}-\theta_{i} \leq$ $\theta, i \in N$.

(H4) $\frac{\theta^{\alpha}\left(m_{1}+m_{2}\right)}{\Gamma(1+\alpha)}\left(\frac{1}{E_{\alpha}\left(m_{1} \theta^{\alpha}\right)}-\frac{m_{2} \theta^{\alpha}}{\Gamma(1+\alpha)}\right)^{-1}<1$.

(H5) $\frac{m_{2} \theta^{\alpha}}{\Gamma(1+\alpha)}+\frac{m_{1} \theta^{\alpha}}{\Gamma(1+\alpha)}\left(1+\frac{m_{2} \theta^{\alpha}}{\Gamma(1+\alpha)}\right) E_{\alpha}\left(m_{1} \theta^{\alpha}\right)<1$, where

$$
m_{1}=\max _{1 \leq i \leq n}\left(a_{i}+L_{i}^{2} \sum_{j=1}^{n}\left|b_{j i}\right|\right), m_{2}=\max _{1 \leq i \leq n}\left(L_{i}^{4} \sum_{j=1}^{n}\left|c_{j i}\right|\right) .
$$

Definition 2.4. The constant vector $x^{*} \in R^{n}$ is a equilibrium point of the neural network system (1), if $x^{*}$ satisfies the equation

$$
-A x^{*}+B f\left(x^{*}\right)+C g\left(x^{*}\right)+I=0 .
$$

Definition 2.5. ([37] Mittag-Leffler stabillity). An equilibrium point $x^{*}$ of the neural network system (1) is said to be Mittag-Leffler stable, if there exist constants $M>0, \lambda>0$ and $b>0$, such that, the solution $x(t)$ with initial value $x(0)=x^{0}$ of system (1) satisfies

$$
\left\|x(t)-x^{*}\right\| \leq\left\{M\left\|x^{0}-x^{*}\right\| E_{\alpha}\left(-\lambda t^{\alpha}\right)\right\}^{b}, t \geq 0 .
$$

In this paper, the deviating argument $\gamma(t)$ is supposed to be discontinuous, thus the right-hand side of system (1) has discontinuities at moments $\theta_{i}, i \in N$. As a result, we define a solution $x(t)$ of system (1) as a continuous function on $R^{+}$, and the derivative $x^{\prime}(t)$ exists at each point $t \in R^{+}$, with the possible exception of the points $\theta_{i}$, $i \in N$, where a one-sided derivative exists. And system (1) is satisfied by $x(t)$ on each interval $\left(\theta_{i}, \theta_{i+1}\right)$ as well.

\subsection{Topological degree properties and Generalized Gronwall-Bellman inequality}

Let $\Omega$ be a nonempty, bounded and open subset of $R^{n}$. The closure of $\Omega$ is denoted by $\bar{\Omega}$, and the boundary of $\Omega$ is denoted by $\partial \Omega$. Lemma 2.4 and Lemma 2.5 demonstrate two properties of the topological degree.

Lemma 2.4. ([38])Let $H(\lambda, x):[0,1] \times \bar{\Omega} \rightarrow R^{n}$ be a continuous homotopy mapping. If $H(\lambda, x)=y$ has no solution in $\partial \Omega$ for $\lambda \in[0,1]$ and $y \in R^{n} \backslash H(\lambda, \partial \Omega)$, then the topological degree $\operatorname{deg}(H(\lambda, x), \Omega, y)$ of $H(\lambda, x)$ is a constant which is independent of $\lambda$. In this case, $\operatorname{deg}(H(0, x), \Omega, y)=\operatorname{deg}(H(1, x), \Omega, y)$.
Lemma 2.5. ([38])Let $H(x): \bar{\Omega} \rightarrow R^{n}$ be a continuous mapping. If $\operatorname{deg}(H(x), \Omega, y) \neq 0$, then there exists at least one solution of $H(x)=y$ in $\Omega$.

Lemma 2.6. ([20])Suppose $x(t) \in R^{n}$ is continuous and differentiable function, $P \in R^{n \times n}$ is a positive definite matrix. Then, for $\alpha \in(0,1)$, the following inequality holds

$$
\frac{1}{2}{ }_{0}^{C} D_{t}^{\alpha} x^{T}(t) P x(t) \leq x^{T}(t) P{ }_{0}^{C} D_{t}^{\alpha} x(t)
$$

Lemma 2.7. ([39]Generalized Gronwall-Bellman inequality). Suppose that $x(t), a(t)$ are non-negative, nondecreasing and local integrable on $0 \leq t<T$, and $g(t)$ is a non-negative, non-decreasing continuous function defined on $0 \leq t<T, g(t) \leq M$ and $M$ is a constant, $\alpha>0$ with

$$
x(t) \leq a(t)+g(t) \int_{0}^{t}(t-s)^{\alpha-1} x(s) d s
$$

on this interval. Then

$$
x(t) \leq a(t) E_{\alpha}\left(g(t) \Gamma(\alpha) t^{\alpha}\right),
$$

where $E_{\alpha}$ is the Mittag-Leffler function.

\subsection{Fractional derivative of integer-order variable up- per limit integral}

In this subsection, we propose a new lemma about fractional derivative of integer-order variable upper limit integral, which will be used in the proof of the main result.

Lemma 2.8. Suppose that $f, g:[0,+\infty) \rightarrow R$ are continuous function, and $g$ is satisfied with $\tau_{1}<g(x)<\tau_{2}$, where $\tau_{1}, \tau_{2}$ are positive constants. Then

$$
\operatorname{Sign}\left(\int_{0}^{t} f(x) d x\right)=\operatorname{Sign}\left(\int_{0}^{t} g(x) f(x) d s\right), \quad t>0,
$$

where Sign(.) is the sign function.

Proof. According to $0<\tau_{1}<g(x)<\tau_{2}$, it follows that the value of $g(x) f(x)$ is between $\tau_{1} f(x)$ and $\tau_{2} f(x)$. Take $t$ as a fixed point. It is easily obtain that $\int_{0}^{t} g(x) f(x) d x$ is between $\int_{0}^{t} \tau_{1} f(x) d x$ and $\int_{0}^{t} \tau_{2} f(x) d x$. Due to $\tau_{1}, \tau_{2}>0$, we can derive that

$$
\operatorname{Sign}\left(\int_{0}^{t} f(x) d x\right)=\operatorname{Sign}\left(\int_{0}^{t} g(x) f(x) d x\right) .
$$




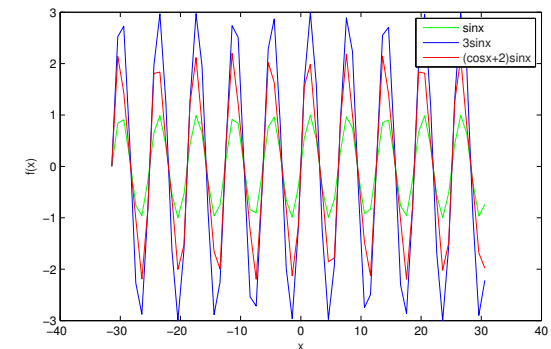

Figure 1: the graph of $\sin x, 3 \sin x$ and $(\cos x+2) \sin x$

Remark 2.1. Let $f(x)=\sin x, g(s)=\cos x+2, x \in$ $[0,+\infty)$. It is easy to check that $1<g(x)<3$. Hence, $g(x)$ satisfies the assumption of Lemma 2.8. In the following, we apply Matlab tool to simulate and verify the validity of Lemma 2.8 .

In Fig.1, we depict the graphs of $\sin x, 3 \sin x$ and $(\cos x+2) \sin x$. It is easy to see that the trace of $(\cos x+$ 2) $\sin x$ is between $\sin x$ and $3 \sin x$.

In Fig.2, we depict the graphs of $\int_{0}^{t} \sin x d x$ and $\int_{0}^{t}(\cos x+2) \sin x d x$. Obviously, the sign of $\int_{0}^{t} \sin x d x$ is the same as the sign of $\int_{0}^{t}(\cos x+2) \sin x d x$.

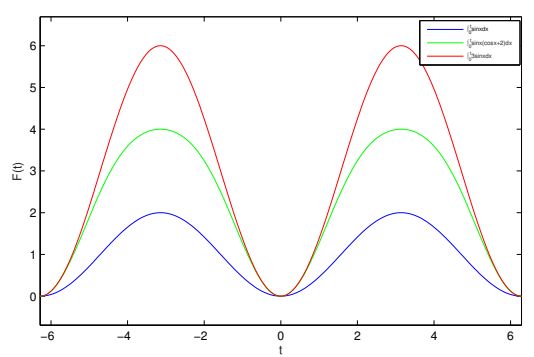

Figure 2: the graph of $\int_{0}^{t} \sin x d x, \int_{0}^{t} 3 \sin x d x$ and $\int_{0}^{t}(\cos x+2) \sin x d x$

Lemma 2.9. Suppose that $z(t)$ and $f(t) \in R$ are continuous functions, and $f(t)$ satisfies

$$
\tau_{1}<\frac{f(z(t))-f(z(s))}{z(t)-z(s)}<\tau_{2},
$$

where $\tau_{1}, \tau_{2}$ are positive constants. Then

$$
{ }_{0}^{C} D_{t}^{\alpha}\left[\int_{0}^{z(s)} f(t) d t\right] \leq f(z(t))_{0}^{C} D_{t}^{\alpha} z(t), \quad \forall \alpha \in(0,1) .
$$

Proof. The inequality (3) is equivalent to

$$
f(z(t))_{0}^{C} D_{t}^{\alpha} z(t)-{ }_{0}^{C} D_{t}^{\alpha}\left[\int_{0}^{z(s)} f(t) d t\right] \geq 0, \quad \forall \alpha \in(0,1) .
$$

According to Definition 2.2, we have

$$
{ }_{0}^{C} D_{t}^{\alpha} z(t)=\frac{1}{\Gamma(1-\alpha)} \int_{0}^{t} \frac{z^{\prime}(s)}{(t-s)^{\alpha}} d s .
$$

$$
\begin{aligned}
{ }_{0}^{C} D_{t}^{\alpha}\left[\int_{0}^{z(s)} f(t) d t\right] & =\frac{1}{\Gamma(1-\alpha)} \int_{0}^{t} \frac{\left(\int_{0}^{z(s)} f(t) d t\right)^{\prime}}{(t-s)^{\alpha}} d s \\
& =\frac{1}{\Gamma(1-\alpha)} \int_{0}^{t} \frac{f(z(s)) z^{\prime}(s)}{(t-s)^{\alpha}} d s .
\end{aligned}
$$

Substituting (5) and (6) into (4), we obtain that

$$
\frac{f(z(t))}{\Gamma(1-\alpha)} \int_{0}^{t} \frac{z^{\prime}(s)}{(t-s)^{\alpha}} d s-\frac{1}{\Gamma(1-\alpha)} \int_{0}^{t} \frac{f(z(s)) z^{\prime}(s)}{(t-s)^{\alpha}} d s \geq 0 .
$$

That is,

$$
-\frac{1}{\Gamma(1-\alpha)} \int_{0}^{t} \frac{f(z(t))-f(z(s))}{(t-s)^{\alpha}}\left(-z^{\prime}(s)\right) d s \geq 0 .
$$

(8) can be changed as

$$
\begin{aligned}
& \frac{1}{\Gamma(1-\alpha)} \int_{0}^{t} \frac{f(z(t))-f(z(s))}{(t-s)^{\alpha}(z(t)-z(s))}(z(t)-z(s))\left(-z^{\prime}(s)\right) d s \\
& \leq 0 .
\end{aligned}
$$

Next, we prove that the inequality

$$
\frac{1}{\Gamma(1-\alpha)} \int_{0}^{t} \frac{(z(t)-z(s))\left(-z^{\prime}(s)\right)}{(t-s)^{\alpha}} d s \leq 0
$$

is true. For convenient, by taking variable transformation $y(s)=z(t)-z(s)$, we discuss

$$
\frac{1}{\Gamma(1-\alpha)} \int_{0}^{t} \frac{y(s) y^{\prime}(s)}{(t-s)^{\alpha}} d s \leq 0 .
$$

Applying integration by parts to (11) yields

$$
\begin{aligned}
& \left.\frac{y^{2}(s)}{2 \Gamma(1-\alpha)(t-s)^{\alpha}}\right|_{s=t}-\left.\frac{y^{2}(s)}{2 \Gamma(1-\alpha)(t-s)^{\alpha}}\right|_{s=0} \\
& -\frac{\alpha}{2 \Gamma(1-\alpha)} \int_{0}^{t} \frac{y^{2}(s)}{(t-s)^{\alpha+1}} d s \leq 0,
\end{aligned}
$$


which is equivalent to

$$
\begin{aligned}
& -\left.\frac{y^{2}(s)}{2 \Gamma(1-\alpha)(t-s)^{\alpha}}\right|_{s=t}+\frac{y^{2}(0)}{2 \Gamma(1-\alpha) t^{\alpha}} \\
& +\frac{\alpha}{2 \Gamma(1-\alpha)} \int_{0}^{t} \frac{y^{2}(s)}{(t-s)^{\alpha+1}} d s \geq 0 .
\end{aligned}
$$

The first term of (13) is singular when $s=t$, we consider the corresponding limitation

$$
\begin{aligned}
\lim _{s \rightarrow t} \frac{y(s) y(s)}{2 \Gamma(1-\alpha)(t-s)^{\alpha}} & =\lim _{s \rightarrow t} \frac{(z(t)-z(s))(z(t)-z(s))}{2 \Gamma(1-\alpha)(t-s)^{\alpha}} \\
& =\lim _{s \rightarrow t} \frac{z^{2}(t)-2 z(t) z(s)+z^{2}(s)}{2 \Gamma(1-\alpha)(t-s)^{\alpha}}
\end{aligned}
$$

By utilizing $L^{\prime} h o ̂ p i t a l$ law to (14), it follows that

$$
\begin{aligned}
& \lim _{s \rightarrow t} \frac{y(s) y(s)}{2 \Gamma(1-\alpha)(t-s)^{\alpha}} \\
= & \lim _{s \rightarrow t} \frac{2 z(t) z^{\prime}(s)-2 z(s) z^{\prime}(s)}{2 \Gamma(1-\alpha) \alpha(t-s)^{\alpha-1}} \\
= & \lim _{s \rightarrow t} \frac{\left[2 z(t) z^{\prime}(s)-2 z(s) z^{\prime}(s)\right](t-s)^{1-\alpha}}{2 \Gamma(1-\alpha) \alpha}=0 .
\end{aligned}
$$

Thus, (13) is reduced to

$$
\frac{y^{2}(0)}{2 \Gamma(1-\alpha) t^{\alpha}}+\frac{\alpha}{2 \Gamma(1-\alpha)} \int_{0}^{t} \frac{y^{2}(s)}{(t-s)^{\alpha+1}} d s \geq 0 .
$$

Obviously, (16) is true. This proves the validity of (10). Noting $0<\tau_{1}<\frac{f(z(t))-f(z(s))}{z(t)-z(s)}<\tau_{2}$, and applying Lemma 2.8, we conclude that (9) is true, which implies

$$
{ }_{0}^{C} D_{t}^{\alpha}\left[\int_{0}^{z(s)} f(t) d t\right] \leq f(z(t))_{0}^{C} D_{t}^{\alpha} z(t), \quad \forall \alpha \in(0,1) .
$$

The proof is completed.

\section{Main results}

\subsection{Existence and uniqueness of solution}

In this subsection, we give the proof for the existence and uniqueness of equilibrium point and solution of the network system (1) on $t \geq 0$.

Theorem 3.1. Suppose that (H1) and (H2) hold, if there exist diagonal matrix $P>0$ and scalar $\varepsilon>0$ such that

$$
\begin{aligned}
\Delta= & P B+(P B)^{T}+\frac{1}{\varepsilon}(P C)(P C)^{T} \\
& +\varepsilon\left(\left(L^{1}\right)^{-1} L^{4}\right)^{T}\left(L^{1}\right)^{-1} L^{4}<0,
\end{aligned}
$$

then the neural network system (1) has a unique equilibrium point.
Proof. (Existence) Set

$$
U(x)=A x-B f(x)-C g(x)-I .
$$

Obviously, $x^{*} \in R^{n}$ is an equilibrium point of system (1), if and only if, $U\left(x^{*}\right)=0$. Construct a mapping $H(\lambda, x): R^{n} \rightarrow R^{n}$ as

$$
H(\lambda, x)=A x-B f(x)-C g(x)-I, \lambda \in[0,1] .
$$

It is easy to check that $H(\lambda, x)$ is a continuous homotopic mapping.

Next, we will construct a convex region $\Omega$, such that $H(\lambda, x) \neq 0, x \in \partial \Omega, \lambda \in[0,1]$.

By the assumption $(\mathrm{H} 1)$, it follows that $x_{i} f_{i}\left(x_{i}\right)=$ $x_{i}\left(f_{i}\left(x_{i}\right)-f_{i}(0)\right)>0$. Combining Lemma 2.3 with assumption (H2) yields

$$
\begin{aligned}
& f^{T}(x) P H(\lambda, x) \\
= & f^{T}(x) P(A x-\lambda(B f(x)+C g(x))-\lambda I) \\
\geq & f^{T}(x)(P A) x-\lambda f^{T}(x)(P I)-\lambda\left[f^{T}(x)(P B) f(x)+\right. \\
& \left.\frac{1}{2 \varepsilon} f^{T}(x)(P C)(P C)^{T} f(x)+\frac{\varepsilon}{2} g^{T}(x) g(x)\right] \\
\geq & f^{T}(x) P(A x-\lambda I)-\frac{\lambda}{2} f^{T}(x)\left[(P B)+(P B)^{T}\right. \\
& \left.\frac{1}{\varepsilon}(P C)(P C)^{T}+\varepsilon\left(\left(L^{1}\right)^{-1} L^{4}\right)^{T}\left(L^{1}\right)^{-1} L^{4}\right] f(x) \\
\geq & f^{T}(x) P(A x-\lambda I) \\
\geq & \sum_{i=1}^{n}\left[\left|f_{i}\left(x_{i}\right)\right| p_{i} a_{i}\left|x_{i}\right|-\left|f_{i}\left(x_{i}\right)\right| p_{i}\left|I_{i}\right|\right] \\
\geq & \sum_{i=1}^{n}\left|f_{i}\left(x_{i}\right)\right| p_{i} a_{i}\left[\left|x_{i}\right|-\left|\frac{I_{i}}{a_{i}}\right|\right] .
\end{aligned}
$$

Under the assumption $\left(H_{2}\right)$, we have

$$
\lim _{\left|x_{i}\right| \rightarrow+\infty} f_{i}\left(x_{i}\right) p_{i} a_{i} x_{i} \geq \lim _{\left|x_{i}\right| \rightarrow+\infty} L_{i}^{1} p_{i} a_{i} x_{i}^{2}=+\infty .
$$

This implies that, for each $i \in\{1,2, \cdots, n\}$,

$$
\lim _{\left|x_{i}\right| \rightarrow+\infty}\left|f_{i}\left(x_{i}\right)\right| p_{i} a_{i}\left(\left|x_{i}\right|-\left|\frac{I_{i}}{a_{i}}\right|\right)=+\infty .
$$

Let

$M_{i}=\sum_{j=1, j \neq i}^{n} \sup \left\{\left|f_{j}\left(x_{j}\right)\right| p_{j} a_{j}\left(\left|x_{j}\right|+\left|\frac{I_{j}}{a_{j}}\right|\right):\left|x_{j}\right| \leq\left|\frac{I_{j}}{a_{j}}\right|\right\}$.

By (19), there exists scalar $m_{i}, m_{i}>\left|\frac{I_{j}}{a_{j}}\right|$, such that for any $\left|x_{i}\right|>m_{i}$, we have

$$
\left|f_{i}\left(x_{i}\right)\right| p_{i} a_{i}\left(\left|x_{i}\right|-\left|\frac{I_{i}}{a_{i}}\right|\right)-M_{i}>0 .
$$


Set $\Omega=\left\{x \in R^{n}:\left|x_{i}\right|<m_{i}+1, i=1,2, \cdots, n\right\}, \Omega$ is an open convex bounded set independent of parameter $\lambda$. If $x \in \partial \Omega$, there must exist a $i_{0} \in\{1,2, \cdots, n\}$ such that $x_{i_{0}}=m_{i_{0}}+1$. For any $\lambda \in[0,1]$, we define following two index sets:

$$
\begin{aligned}
& \Theta_{+}=\left\{i \in\{1,2, \cdots, n\}:\left|x_{i}\right| \geq\left|\frac{I_{i}}{a_{i}}\right|\right\}, \\
& \Theta_{-}=\left\{i \in\{1,2, \cdots, n\}:\left|x_{i}\right|<\left|\frac{I_{i}}{a_{i}}\right|\right\}=\{1,2, \cdots, n\} \backslash \Theta_{+} .
\end{aligned}
$$

By the expression of $M_{i}$ and (20), for any $x \in \partial \Omega$ and $\lambda \in[0,1]$, it follows that

$$
\begin{aligned}
& f^{T}(x) P H(\lambda, x) \\
\geq & \sum_{i=1}^{n}\left|f_{i}\left(x_{i}\right)\right| p_{i} a_{i}\left(\left|x_{i}\right|-\left|\frac{I_{i}}{a_{i}}\right|\right) \\
= & \sum_{i \in \Theta_{+} \backslash\left\{i_{0}\right\}}\left|f_{i}\left(x_{i}\right)\right| p_{i} a_{i}\left(\left|x_{i}\right|-\left|\frac{I_{i}}{a_{i}}\right|\right) \\
& +\left|f_{i_{0}}\left(x_{i_{0}}\right)\right| p_{i_{0}} a_{i_{0}}\left(\left|x_{i_{0}}\right|-\left|\frac{I_{i_{0}}}{a_{i_{0}}}\right|\right) \\
& +\sum_{i \in \Theta_{-}}\left|f_{i}\left(x_{i}\right)\right| p_{i} a_{i}\left(\left|x_{i}\right|-\left|\frac{I_{i}}{a_{i}}\right|\right) \\
> & 0+\left|f_{i_{0}}\left(x_{i_{0}}\right)\right| p_{i_{0}} a_{i_{0}}\left(\left|x_{i_{0}}\right|-\left|\frac{I_{i_{0}}}{a_{i_{0}}}\right|\right)-M_{i_{0}}
\end{aligned}
$$$$
>0 \text {. }
$$

This implies that $H(\lambda, x) \neq 0$ for any $x \in \partial \Omega, \lambda \in[0,1]$.

By Lemma 2.4, we obtain that $\operatorname{deg}(H(1, x), \Omega, 0)=$ $\operatorname{deg}(H(0, x), \Omega, 0)$, i.e., $\operatorname{deg}(U(x), \Omega, 0)=\operatorname{deg}(A x, \Omega, 0)$ $=\operatorname{Sgn}|A| \neq 0$, where $|A|$ is the determinant of $\mathrm{A}$. By Lemma 2.5, $U(x)=0$ has at least one solution in $\Omega$. This implies that the network system (1) has at least one equilibrium point in $\Omega$.

(Uniqueness) Suppose that $x_{1}^{*}$ and $x_{2}^{*}$ are two different equilibrium points of the system (1). Then

$$
A\left(x_{1}^{*}-x_{2}^{*}\right)=B\left[f\left(x_{1}^{*}\right)-f\left(x_{2}^{*}\right)\right]+C\left[g\left(x_{1}^{*}\right)-g\left(x_{2}^{*}\right)\right]
$$

Multiplying by $2\left[f\left(x_{1}^{*}\right)-f\left(x_{2}^{*}\right)\right]^{T} P$, we obtain that

$$
\begin{aligned}
& 0<2\left[f\left(x_{1}^{*}\right)-f\left(x_{2}^{*}\right)\right]^{T} P A\left(x_{1}^{*}-x_{2}^{*}\right) \\
= & 2\left[f\left(x_{1}^{*}\right)-f\left(x_{2}^{*}\right)\right]^{T} P\left(B\left[f\left(x_{1}^{*}\right)-f\left(x_{2}^{*}\right)\right]+C\left[g\left(x_{1}^{*}\right)-g\left(x_{2}^{*}\right)\right]\right) \\
\leq & {\left[f\left(x_{1}^{*}\right)-f\left(x_{2}^{*}\right)\right]^{T}\left\{P B+(P B)^{T}+\frac{1}{\varepsilon}(P C)(P C)^{T}\right.} \\
& \left.+\varepsilon\left(\left(L^{1}\right)^{-1} L^{4}\right)^{T}\left(L^{1}\right)^{-1} L^{4}\right\}\left[f\left(x_{1}^{*}\right)-f\left(x_{2}^{*}\right)\right] \\
\leq & {\left[f\left(x_{1}^{*}\right)-f\left(x_{2}^{*}\right)\right]^{T} \Delta\left[f\left(x_{1}^{*}\right)-f\left(x_{2}^{*}\right)\right] } \\
< & 0 .
\end{aligned}
$$

This is a contradiction. The above discussion shows that system (1) has a unique equilibrium point. This completes the proof.
Theorem 3.2. Suppose that assumptions (H1)-(H4) are established. Then, for any $\left(t_{0}, x^{0}\right) \in R^{+} \times R^{n}$, there exists a unique solution $x(t)=x\left(t, t_{0}, x^{0}\right)=\left(x_{1}(t), \cdots, x_{n}(t)\right)^{T}$ on $\left[t_{0},+\infty\right)$ for system $(1)$, such that $x\left(t_{0}\right)=x^{0}$.

Proof. (Existence) Fix $k \in N$. In generally, we assume that $\theta_{k} \leq \zeta_{k}<t_{0} \leq \theta_{k+1}$. First of all, for every $\left(t_{0}, x^{0}\right) \in R^{+} \times R^{n}$, we shall prove that there exists a solution $x(t)=x\left(t, t_{0}, x^{0}\right)=\left(x_{1}(t), \cdots, x_{n}(t)\right)$ of (1) under the initial value $x\left(t_{0}\right)=x^{0}=\left(x_{1}^{0}, \cdots, x_{n}^{0}\right)^{T}$.

By Lemma 2.1, the fractional order differential equation (2) is equivalent to the corresponding fractionalorder integral equation

$$
\begin{aligned}
x_{i}(t)= & x_{i}^{0}+{ }_{t_{0}}^{R} I_{t}^{\alpha}\left[-a_{i} x_{i}(s)+\sum_{j=1}^{n} b_{i j} f_{j}\left(x_{j}(s)\right)\right. \\
& \left.+\sum_{j=1}^{n} c_{i j} g_{j}\left(x_{j}\left(\zeta_{k}\right)\right)+I_{i}\right] .
\end{aligned}
$$

Define a norm $\|x(t)\|_{0}=\max _{\left[\zeta_{k}, t_{0}\right]}\|x(t)\|$ and construct the sequence of iterations $x_{i}^{m}(t), x_{i}^{0}(t) \equiv x_{i}^{0}, i=$ $1,2, \cdots, n, m \geq 0$ as

$$
\begin{aligned}
x_{i}^{m+1}(t)= & x_{i}^{0}+{ }_{t_{0}}^{R} I_{t}^{\alpha}\left[-a_{i} x_{i}^{m}(s)+\sum_{j=1}^{n} b_{i j} f_{j}\left(x_{j}^{m}(s)\right)\right. \\
& \left.+\sum_{j=1}^{n} c_{i j} g_{j}\left(x_{j}^{m}\left(\zeta_{k}\right)\right)+I_{i}\right] .
\end{aligned}
$$

It follows from $(\mathrm{H} 2)$ that

$$
\begin{aligned}
& \left\|x^{m+1}(t)-x^{m}(t)\right\| \\
\leq & \frac{1}{\Gamma(\alpha)} \sum_{i=1}^{n}\left\{\int _ { t _ { 0 } } ^ { t } ( t - s ) ^ { \alpha - 1 } \left[\left(a_{i}+L_{i}^{2} \sum_{j=1}^{n}\left|b_{j i}\right|\right)\left|x_{i}^{m}(s)-x_{i}^{m-1}(s)\right|\right.\right. \\
& \left.\left.+\left(L_{i}^{4} \sum_{j=1}^{n}\left|c_{j i}\right|\right)\left|x_{i}^{m}\left(\zeta_{k}\right)-x_{i}^{m-1}\left(\zeta_{k}\right)\right|\right] d s\right\} .
\end{aligned}
$$

By the assumption (H3) and (H4), we obtain that

$$
\begin{aligned}
& \left\|x^{m+1}(t)-x^{m}(t)\right\|_{0} \\
\leq & \frac{1}{\Gamma(\alpha)} \sum_{i=1}^{n}\left\{\int _ { t _ { 0 } } ^ { t } ( t - s ) ^ { \alpha - 1 } \left[m_{1}\left|x_{i}^{m}(s)-x_{i}^{m-1}(s)\right|\right.\right. \\
& \left.\left.+m_{2}\left|x_{i}^{m}\left(\zeta_{k}\right)-x_{i}^{m-1}\left(\zeta_{k}\right)\right|\right] d s\right\} \\
\leq & \frac{1}{\Gamma(\alpha)}\left\{\int _ { t _ { 0 } } ^ { t } ( t - s ) ^ { \alpha - 1 } \left[m_{1}\left\|x^{m}(s)-x^{m-1}(s)\right\|\right.\right. \\
& \left.\left.+m_{2}\left\|x^{m}\left(\zeta_{k}\right)-x^{m-1}\left(\zeta_{k}\right)\right\|\right] d s\right\} \\
\leq & \frac{\theta^{\alpha}}{\Gamma(1+\alpha)}\left(m_{1}+m_{2}\right)\left\|x^{m}(t)-x^{m-1}(t)\right\|_{0} .
\end{aligned}
$$


It is easy to get that

$$
\begin{aligned}
\left\|x^{m+1}(t)-x^{m}(t)\right\|_{0} & \leq\left(\frac{\left(m_{1}+m_{2}\right) \theta^{\alpha}}{\Gamma(1+\alpha)}\right)^{m}\left\|x^{1}(t)-x^{0}(t)\right\| \\
& \leq\left(\frac{\left(m_{1}+m_{2}\right) \theta^{\alpha}}{\Gamma(1+\alpha)}\right)^{m} \tau,
\end{aligned}
$$

where

$$
\tau=\frac{\theta^{\alpha}}{\Gamma(1+\alpha)}\left[\left(m_{1}+m_{2}\right)\left\|x^{0}\right\|+\sum_{i=1}^{n} I_{i}\right] .
$$

Thus, we conclude that there exists a solution $x(t)=$ $x\left(t, t_{0}, x\left(t_{0}\right)\right)$ for system (2) on $\left[\zeta_{k}, t_{0}\right]$. By the assumption (H2), it follows that $x(t)$ can be continued to $\theta_{k+1}$. The above operation can be done once again on interval $\left(\theta_{k+1}, \theta_{k+2}\right]$. By the mathematical induction, we conclude that there exists a solution $x\left(t, t_{0}, x^{0}\right)$ with $x\left(t_{0}\right)=$ $x^{0}$ on $\left[t_{0},+\infty\right)$ for system (1).

(Uniqueness) First, let us discuss the $\theta_{k} \leq t_{0} \leq \theta_{k+1}$. Define by $x^{1}(t)=x\left(t, t_{0}, x^{1}\right), x^{2}(t)=x\left(t, t_{0}, x^{2}\right)$ the solution of system (1).

By reduction to absurdity, for every $t \in\left[\theta_{k}, \theta_{k+1}\right]$, we suppose that $x^{1}=\left(x_{1}^{1}, \cdots, x_{n}^{1}\right), x^{2}=\left(x_{1}^{2}, \cdots, x_{n}^{2}\right), x^{1} \neq$ $x^{2}$, which means that $x^{1}(t) \neq x^{2}(t)$. Then

$$
\begin{aligned}
& \left\|x^{1}(t)-x^{2}(t)\right\| \\
& \leq\left\|x^{1}-x^{2}\right\|+\frac{1}{\Gamma(\alpha)} \sum_{i=1}^{n}\left\{\int_{t_{0}}^{t}(t-s)^{\alpha-1}\right. \\
& \\
& {\left[\left(a_{i}+L_{i}^{2} \sum_{j=1}^{n}\left|b_{j i}\right|\right)\left|x_{i}^{1}(s)-x_{i}^{2}(s)\right|\right.} \\
& \left.\left.+\left(L_{i}^{4} \sum_{j=1}^{n}\left|c_{j i}\right|\right)\left|x_{i}^{1}\left(\zeta_{k}\right)-x_{i}^{2}\left(\zeta_{k}\right)\right|\right] d s\right\} \\
& \leq\left(\left\|x^{1}-x^{2}\right\|+\frac{\theta^{\alpha}}{\Gamma(1+\alpha)} m_{2}\left\|x^{1}\left(\zeta_{k}\right)-x^{2}\left(\zeta_{k}\right)\right\|\right) \\
& \quad+\frac{1}{\Gamma(\alpha)} \int_{t_{0}}^{t}(t-s)^{\alpha-1} m_{1}\left\|x^{1}(s)-x^{2}(s)\right\| d s .
\end{aligned}
$$

By Lemma 2.7, it follows that

$$
\begin{aligned}
& \left\|x^{1}(t)-x^{2}(t)\right\| \\
\leq & \left(\left\|x^{1}-x^{2}\right\|+\frac{\theta^{\alpha}}{\Gamma(1+\alpha)} m_{2}\left\|x^{1}\left(\zeta_{k}\right)-x^{2}\left(\zeta_{k}\right)\right\|\right) E_{\alpha}\left(m_{1} \theta^{\alpha}\right) .
\end{aligned}
$$

In particular,

$$
\begin{aligned}
& \left\|x^{1}\left(\zeta_{k}\right)-x^{2}\left(\zeta_{k}\right)\right\| \\
\leq & \left(\left\|x^{1}-x^{2}\right\|+\frac{\theta^{\alpha}}{\Gamma(1+\alpha)} m_{2}\left\|x^{1}\left(\zeta_{k}\right)-x^{2}\left(\zeta_{k}\right)\right\|\right) E_{\alpha}\left(m_{1} \theta^{\alpha}\right) .
\end{aligned}
$$

Therefore,

$$
\left\|x^{1}(t)-x^{2}(t)\right\| \leq\left(\frac{1}{E_{\alpha}\left(m_{1} \theta^{\alpha}\right)}-\frac{m_{2} \theta^{\alpha}}{\Gamma(1+\alpha)}\right)^{-1}\left\|x^{1}-x^{2}\right\| .
$$

On the other hand, assume opposite that there exists $t \in$ $\left[\theta_{k}, \theta_{k+1}\right]$ such that $x^{1}(t)=x^{2}(t)$. Then, we have

$$
\begin{aligned}
& \left\|x^{1}-x^{2}\right\| \\
= & \frac{1}{\Gamma(\alpha)} \sum_{i=1}^{n}\left\{\int _ { t _ { 0 } } ^ { t } ( t - s ) ^ { \alpha - 1 } \left[-a_{i}\left(x_{i}^{2}(s)-x_{i}^{1}(s)\right)\right.\right. \\
& +\sum_{j=1}^{n} b_{i j}\left[f _ { j } \left(x_{j}^{2}(s)-f_{j}\left(x_{j}^{1}(s)\right)\right.\right. \\
& +\sum_{j=1}^{n} c_{i j}\left[g_{j}\left(x_{j}^{2}\left(\zeta_{k}\right)-g_{j}\left(x_{j}^{1}\left(\zeta_{k}\right)\right)\right] d s\right\} \\
= & \frac{1}{\Gamma(\alpha)} \sum_{i=1}^{n}\left\{\int_{t_{0}}^{t}(t-s)^{\alpha-1}\left(a_{i}+L_{i}^{2} \sum_{j=1}^{n}\left|b_{j i}\right|\right)\right. \\
& \left.\times\left|x_{i}^{1}(s)-x_{i}^{2}(s)\right|+\left(L_{i}^{4} \sum_{j=1}^{n}\left|c_{j i}\right|\right)\left|x_{i}^{1}\left(\zeta_{k}\right)-x_{i}^{2}\left(\zeta_{k}\right)\right| d s\right\} \\
\leq & \frac{\theta^{\alpha}}{\Gamma(1+\alpha)} m_{2}\left\|x^{1}\left(\zeta_{k}\right)-x^{2}\left(\zeta_{k}\right)\right\| \\
& +\frac{1}{\Gamma(\alpha)} \int_{t_{0}}^{t}(t-s)^{\alpha-1} m_{1}\left\|x^{1}(s)-x^{2}(s)\right\| d s .
\end{aligned}
$$

By substituting (21) into (22) and applying assumption (H4), we obtain that

$$
\begin{aligned}
\left\|x^{1}-x^{2}\right\| & \leq \frac{\theta^{\alpha}\left(m_{1}+m_{2}\right)}{\Gamma(1+\alpha)}\left(\frac{1}{E_{\alpha}\left(m_{1} \theta^{\alpha}\right)}-\frac{m_{2} \theta^{\alpha}}{\Gamma(1+\alpha)}\right)^{-1}\left\|x^{1}-x^{2}\right\| \\
& <\left\|x^{1}-x^{2}\right\| .
\end{aligned}
$$

This is a contradiction. This proves that the uniqueness of solution for $t \in\left[\theta_{k}, \theta_{k+1}\right]$. The extension of the unique solution on $R^{+}$is obvious. The proof is completed.

By substituting $x(t)=y(t)+x^{*}$, system (2) can be simplified as

$$
\begin{aligned}
{ }_{0}^{C} D_{t}^{\alpha} y_{i}(t)= & -a_{i} y_{i}(t)+\sum_{j=1}^{n} b_{i j} \varphi_{j}\left(y_{j}(t)\right) \\
& +\sum_{j=1}^{n} c_{i j} \psi_{j}\left(y_{j}(\gamma(t))\right), \quad i=1, \cdots, n .
\end{aligned}
$$

where $\varphi_{j}\left(y_{j}(t)\right)=f_{j}\left(y_{j}(t)+x_{j}^{*}\right)-f_{j}\left(x_{j}^{*}\right), \psi_{j}\left(y_{j}(\gamma(t))\right)=$ $g_{j}\left(y_{j}\left(\gamma(t)+x_{j}^{*}\right)\right)-g_{j}\left(x_{j}^{*}\right)$ with $\varphi_{j}(0)=0, \psi_{j}(0)=0$. It is 
easy to see that the equilibrium point of system (24) is the original point. The stability of system (24) is equal to the stability of equilibrium $x^{*}$ of system (2). Therefore, in the following, we only discuss the stability of the zero solution of system (24).

First of all, we address the following lemma, which is one of the important auxiliary results in this paper.

Lemma 3.1. Suppose that assumptions $(H 1)-(H 5)$ are satisfied. Let $y(t)=\left(y_{1}(t), \cdots, y_{n}(t)\right)^{T}$ be a solution of system (24). Then, the following inequality

$$
\|y(\gamma(t))\| \leq \eta\|y(t)\|
$$

holds for $t \in R^{+}$, where

$\eta=\left(1-\frac{m_{2} \theta^{\alpha}}{\Gamma(1+\alpha)}-\frac{m_{1} \theta^{\alpha}}{\Gamma(1+\alpha)}\left(1+\frac{m_{2} \theta^{\alpha}}{\Gamma(1+\alpha)}\right) E_{\alpha}\left(m_{1} \theta^{\alpha}\right)\right)^{-1}$.

Proof. Fix $k \in N$. Then, for $t \in\left[\theta_{k}, \theta_{k+1}\right)$, we have

$$
\begin{aligned}
y_{i}(t)= & y_{i}\left(\zeta_{k}\right)+{ }_{\zeta_{k}}^{R} I_{t}^{\alpha}\left[-a_{i} y_{i}(s)+\sum_{j=1}^{n} b_{i j} \varphi_{j}\left(y_{j}(s)\right)\right. \\
& \left.+\sum_{j=1}^{n} c_{i j} \psi_{j}\left(y_{j}\left(\zeta_{k}\right)\right)\right]
\end{aligned}
$$

where $\gamma(t)=\zeta_{k}$, if $t \in\left[\theta_{k}, \theta_{k+1}\right), t \in R^{+}$. Taking absolute value of both sides of above expression for each $i=$ $1,2, \cdots, n$, we obtain that

$$
\begin{aligned}
\|y(t)\| \leq & \left\|y\left(\zeta_{k}\right)\right\|+\frac{1}{\Gamma(\alpha)} \sum_{i=1}^{n}\left\{\int _ { \zeta _ { k } } ^ { t } ( t - s ) ^ { \alpha - 1 } \left[a_{i}\left|y_{i}(s)\right|\right.\right. \\
& \left.\left.+\sum_{j=1}^{n}\left|b_{i j}\left\|\varphi_{j}\left(y_{j}(s)\right)\left|+\sum_{j=1}^{n}\right| c_{i j}\right\| \psi_{j}\left(y_{j}\left(\zeta_{k}\right)\right)\right|\right] d s\right\} \\
\leq & \left\|y\left(\zeta_{k}\right)\right\|+\frac{1}{\Gamma(\alpha)}\left\{\int _ { \zeta _ { k } } ^ { t } ( t - s ) ^ { \alpha - 1 } \left[\sum_{i=1}^{n}\left\{a_{i}+L_{i}^{2} \sum_{j=1}^{n}\left|b_{j i}\right|\right)\right.\right. \\
& \left.\left.\left|y_{i}(s)\right|+\sum_{i=1}^{n}\left(L_{i}^{4} \sum_{j=1}^{n}\left|c_{j i}\right|\right)\left|y_{i}\left(\zeta_{k}\right)\right|\right] d s\right\} \\
\leq & \left\|y\left(\zeta_{k}\right)\right\|+\frac{1}{\Gamma(\alpha)}\left\{\int _ { \zeta _ { k } } ^ { t } ( t - s ) ^ { \alpha - 1 } \left[m_{1}\|y(s)\|\right.\right. \\
& \left.\left.+m_{2}\left\|y\left(\zeta_{k}\right)\right\|\right] d s\right\} \\
= & \left\|y\left(\zeta_{k}\right)\right\|+\frac{m_{2}\left\|y\left(\zeta_{k}\right)\right\|}{\Gamma(\alpha)} \times \frac{\left(t-\zeta_{k}\right)^{\alpha}}{\alpha} \\
& +\frac{m_{1}}{\Gamma(\alpha)} \int_{\zeta_{k}}^{t}(t-s)^{\alpha-1}\|y(s)\| d s \\
= & \left(1+\frac{m_{2} \theta^{\alpha}}{\Gamma(1+\alpha)}\right)\left\|y\left(\zeta_{k}\right)\right\| \\
& +\frac{m_{1}}{\Gamma(\alpha)} \int_{\zeta_{k}}^{t}(t-s)^{\alpha-1}\|y(s)\| d s . \\
&
\end{aligned}
$$

By applying Lemma 2.7, it yields

$$
\|y(t)\| \leq\left(1+\frac{m_{2} \theta^{\alpha}}{\Gamma(1+\alpha)}\right)\left\|y\left(\zeta_{k}\right)\right\| E_{\alpha}\left(m_{1} t^{\alpha}\right) .
$$

Furthermore, for $t \in\left[\theta_{k}, \theta_{k+1}\right)$, we have

$$
\begin{aligned}
\left\|y\left(\zeta_{k}\right)\right\| \leq & \|y(t)\|+\frac{m_{2}\left\|y\left(\zeta_{k}\right)\right\|}{\Gamma(\alpha)} \times \frac{\left(t-\zeta_{k}\right)^{\alpha}}{\alpha} \\
& +\frac{m_{1}}{\Gamma(\alpha)} \int_{\zeta_{k}}^{t}(t-s)^{\alpha-1}\|y(s)\| d s \\
\leq & \|y(t)\|+\frac{m_{2} \theta^{\alpha}\left\|y\left(\zeta_{k}\right)\right\|}{\Gamma(1+\alpha)} \\
& +\frac{m_{1}}{\Gamma(\alpha)} \int_{\zeta_{k}}^{t}(t-s)^{\alpha-1}\|y(s)\| d s .
\end{aligned}
$$

Combining (26) with (27), we get

$$
\begin{aligned}
\left\|y\left(\zeta_{k}\right)\right\| \leq & \|y(t)\|+\frac{m_{2} \theta^{\alpha}\left\|y\left(\zeta_{k}\right)\right\|}{\Gamma(1+\alpha)}+\frac{m_{1}}{\Gamma(\alpha)} \\
& \times \int_{\zeta_{k}}^{t}(t-s)^{\alpha-1}\left(1+\frac{m_{2} \theta^{\alpha}}{\Gamma(1+\alpha)}\right)\left\|y\left(\zeta_{k}\right)\right\| E_{\alpha}\left(m_{1} t^{\alpha}\right) d s \\
\leq & \|y(t)\|+\frac{m_{2} \theta^{\alpha}\left\|y\left(\zeta_{k}\right)\right\|}{\Gamma(1+\alpha)}+\frac{m_{1}}{\Gamma(\alpha)} \times \frac{\theta^{\alpha}}{\alpha} \\
& \times\left(1+\frac{m_{2} \theta^{\alpha}}{\Gamma(1+\alpha)}\right)\left\|y\left(\zeta_{k}\right)\right\| E_{\alpha}\left(m_{1} t^{\alpha}\right) \\
\leq & \|y(t)\|+\left[\frac{m_{2} \theta^{\alpha}}{\Gamma(1+\alpha)}+\frac{m_{1} \theta^{\alpha}}{\Gamma(1+\alpha)}\right. \\
& \left.\times\left(1+\frac{m_{2} \theta^{\alpha}}{\Gamma(1+\alpha)}\right) E_{\alpha}\left(m_{1} t^{\alpha}\right)\right]\left\|y\left(\zeta_{k}\right)\right\| .
\end{aligned}
$$

Therefore, by assumption (H5), it follows that

$\left\|y\left(\zeta_{k}\right)\right\| \leq \eta\|y(t)\|, \quad t \in\left[\theta_{k}, \theta_{k+1}\right)$,

where $\eta=\left(1-\frac{m_{2} \theta^{\alpha}}{\Gamma(1+\alpha)}-\frac{m_{1} \theta^{\alpha}}{\Gamma(1+\alpha)}\left(1+\frac{m_{2} \theta^{\alpha}}{\Gamma(1+\alpha)}\right) E_{\alpha}\left(m_{1} \theta^{\alpha}\right)\right)^{-1}$. This implies that (25) holds for $t \in R^{+}$. The proof is completed.

\subsection{Stability analysis}

In this subsection, we establish some criteria for global Mittag-Leffler stability of the network system (1).

Theorem 3.3. Supposed that assumptions (H1)-(H5) hold. If there exist a scalar $\varepsilon>0$ and matrix $P=$ $\operatorname{diag}\left(p_{1}, p_{2}, \cdots, p_{n}\right)>0$, such that

$$
\begin{aligned}
\widetilde{\Delta}= & P B+(P B)^{T}+\frac{1}{\varepsilon}(P C)(P C)^{T} \\
& +\varepsilon \eta^{2}\left(\left(L^{1}\right)^{-1} L^{4}\right)^{T}\left(L^{1}\right)^{-1} L^{4}<0,
\end{aligned}
$$

then the network system (1) is globally Mittag-Leffler stable. 
Proof. Consider Lur'e Postnikov type Lyapunov functional

$$
V(t)=\frac{1}{2} y^{T}(t) P y(t)+\beta \sum_{i=1}^{n} p_{i} \int_{y_{i}(\gamma(t))}^{y_{i}(t)} \varphi_{i}(s) d s .
$$

Taking Caputo's derivative of order $\alpha$ of $V(t)$ at $t$ along the trajectories of system (24), and applying Lemma 2.6 and 2.9, we have

$$
\begin{aligned}
{ }_{0}^{C} D_{t}^{\alpha} V(t) \leq & y^{T}(t) P_{0}^{C} D_{t}^{\alpha} y(t)+\beta \sum_{i=1}^{n} p_{i} \varphi_{i}\left(y_{i}(t)\right)_{0}^{C} D_{t}^{\alpha} y_{i}(t) \\
\leq & y^{T}(t) P_{0}^{C} D_{t}^{\alpha} y(t)+\beta \sum_{i=1}^{n} p_{i} \varphi_{i}\left(y_{i}(t)\right) \times\left[-a_{i} y_{i}(t)\right. \\
& \left.+\sum_{j=1}^{n} b_{i j} \varphi_{j}\left(y_{j}(t)\right)+\sum_{j=1}^{n} c_{i j} \psi_{j}\left(y_{j}(t)\right)\right] \\
= & y^{T}(t) P_{0}^{C} D_{t}^{\alpha} y(t)+\beta\left[\varphi^{T}(y(t))(-P A) y(t)\right) \\
& \left.+\varphi^{T}(y(t))(P B) \varphi(y(t))+\varphi^{T}(y(t))(P C) \psi(y(\gamma(t)))\right] \\
= & -\frac{1}{2} y^{T}(t)\left(P A+(P A)^{T}\right) y(t)+y^{T}(t)(P B) \varphi(y(t) \\
& +y^{T}(t)(P C) \psi(y(\gamma(t)))+\beta\left[\varphi^{T}(y(t))(-P A) y(t)\right) \\
& +\frac{1}{2} \varphi^{T}(y(t))\left(P B+(P B)^{T}\right) \varphi(y(t)) \\
& \left.+\varphi^{T}(y(t))(P C) \psi(y(\gamma(t)))\right] .
\end{aligned}
$$

Applying Lemma 2.3 yields

$$
\begin{aligned}
y^{T}(t) P B \varphi(y(t)) \leq & \frac{1}{2 \varepsilon} y^{T}(t)(P B)(P B)^{T} y(t) \\
& +\frac{\varepsilon}{2} \varphi^{T}(y(t)) \varphi(y(t)) .
\end{aligned}
$$

$$
\begin{aligned}
y^{T}(t)(P C) \psi(y(\gamma(t))) \leq & \frac{1}{2 \varepsilon} y^{T}(t)(P C)(P C)^{T} y(t) \\
& +\frac{\varepsilon}{2} \psi^{T}(y(\gamma(t))) \psi(y(\gamma(t))) . \\
\varphi^{T}(y(t))(P C) \psi(y(\gamma(t))) \leq & \frac{1}{2 \varepsilon} \varphi^{T}(y(t))(P C)(P C)^{T} \varphi(y(t)) \\
& +\frac{\varepsilon}{2} \psi^{T}(y(\gamma(t))) \psi(y(\gamma(t))) .
\end{aligned}
$$

According to assumption (H2), it follows that

$$
\begin{aligned}
& \varphi^{T}(y(t)) \varphi(y(t)) \\
= & {\left[f\left(y(t)+x^{*}\right)-f\left(x^{*}\right)\right]^{T}\left[f\left(y(t)+x^{*}\right)-f\left(x^{*}\right)\right] } \\
\leq & y^{T}(t)\left(L^{2}\right)^{T} L^{2} y(t) .
\end{aligned}
$$

$$
\psi^{T}(y(\gamma(t))) \psi(y(\gamma(t))) \leq y^{T}(\gamma(t))\left(L^{4}\right)^{T} L^{4} y(\gamma(t)) .
$$

By Lemma 3.1, we have

$$
y^{T}(\gamma(t)) y(\gamma(t)) \leq \eta^{2} y^{T}(t) y(t) .
$$

Substituting (29)-(34) into (28), we have

$$
\begin{aligned}
& { }_{0}^{C} D_{t}^{\alpha} V(t) \\
\leq & -\frac{1}{2} y^{T}(t)(P A) y(t)+\frac{1}{2} y^{T}(t)\left(\frac{1}{\varepsilon}(P B)(P B)^{T}\right. \\
& \left.+\varepsilon\left(L^{2}\right)^{T} L^{2}+\frac{1}{\varepsilon}(P C)(P C)^{T}+\varepsilon \eta^{2}\left(L^{4}\right)^{T} L^{4}\right) y(t) \\
& \left.+\beta \varphi^{T}(y(t))(-P A) y(t)\right)+\frac{1}{2} \beta y^{T}(t)\left(L^{2}\right)^{T}\left(P B+(P B)^{T}\right. \\
& \left.+\frac{1}{\varepsilon}(P C)(P C)^{T}+\varepsilon \eta^{2}\left(\left(L^{1}\right)^{-1} L^{4}\right)^{T}\left(L^{1}\right)^{-1} L^{4}\right) L^{2} y(t) .
\end{aligned}
$$

Let $\Sigma=\frac{1}{\varepsilon}(P B)(P B)^{T}+\varepsilon\left(L^{2}\right)^{T} L^{2}+\frac{1}{\varepsilon}(P C)(P C)^{T}+$ $\varepsilon \eta^{2}\left(L^{4}\right)^{T} L^{4}$. Obviously, $\Sigma>0$.

Take $\beta>\frac{\lambda_{\max }(\Sigma)}{\lambda_{\min }\left(-\left(L^{2}\right)^{T} \widetilde{\Delta} L^{2}\right)}>0$. Then

$$
\begin{aligned}
& { }_{0}^{C} D_{t}^{\alpha} V(t) \\
\leq & \left.-\frac{1}{2} y^{T}(t)(P A) y(t)+\beta \varphi^{T}(y(t))(-P A) y(t)\right) \\
& +\frac{1}{2} y^{T}(t) \Sigma y(t)+\frac{1}{2} \beta y^{T}(t)\left(\left(L^{2}\right)^{T} \widetilde{\Delta} L^{2}\right) y(t) \\
\leq & \left.-\frac{1}{2} y^{T}(t)(P A) y(t)+\beta \varphi^{T}(y(t))(-P A) y(t)\right) \\
\leq & -\lambda_{\min }(A)\left(\frac{1}{2} y^{T}(t) P y(t)+\beta \sum_{i=1}^{n} p_{i} \int_{y_{i}(\gamma(t))}^{y_{i}(t)} \varphi_{i}(s) d s\right) .
\end{aligned}
$$

This implies that

$$
{ }_{0}^{C} D_{t}^{\alpha} V(t) \leq-\lambda V(t),
$$

where $\lambda=\lambda_{\min }(A)$. By Lemma 2.2, from (35), we get

$$
V(t) \leq V(0) E_{\alpha}\left(-\lambda t^{\alpha}\right), \quad t>0 .
$$

Noting that

$$
\begin{aligned}
V(t) & =\frac{1}{2} y^{T}(t) P y(t)+\beta \sum_{i=1}^{n} p_{i} \int_{y_{i}(\gamma(t))}^{y_{i}(t)} \varphi_{i}(s) d s \\
& \geq \frac{1}{2} y^{T}(t) P y(t) \\
& \geq \frac{1}{2} \lambda_{\min }(P)\|y(t)\|^{2},
\end{aligned}
$$


we have

$$
\|y(t)\|^{2} \leq \frac{2}{\lambda_{\min }(P)} V(0) E_{\alpha}\left(-\lambda t^{\alpha}\right), \quad t>0 .
$$

Hence, we obtain that

$$
\begin{aligned}
& \left\|x(t)-x^{*}\right\|^{2} \\
\leq & \frac{2}{\lambda_{\min }(P)}\left(\frac{\lambda_{\max }(P)}{2}\left\|x(0)-x^{*}\right\|^{2}\right. \\
& \left.+\beta \sum_{i=1}^{n} p_{i} \int_{0}^{x(0)-x^{*}} \varphi_{i}(s) d s\right) E_{\alpha}\left(-\lambda t^{\alpha}\right) \\
\leq & \frac{2}{\lambda_{\min }(P)}\left(\frac{\lambda_{\max }(P)}{2}\left\|x(0)-x^{*}\right\|^{2}+\right. \\
& \left.\beta \lambda_{\max }(P) \varphi^{T}\left(x(0)-x^{*}\right)\left(x(0)-x^{*}\right)\right) E_{\alpha}\left(-\lambda t^{\alpha}\right) \\
\leq & \frac{\lambda_{\max }(P)}{\lambda_{\min }(P)}\left(1+2 \beta L^{4}\right)\left\|x(0)-x^{*}\right\|^{2} E_{\alpha}\left(-\lambda t^{\alpha}\right),
\end{aligned}
$$

i.e.,

$$
\left\|x(t)-x^{*}\right\| \leq \sqrt{\frac{\lambda_{\max }(P)}{\lambda_{\min }(P)}}\left\|x(0)-x^{*}\right\|\left(E_{\alpha}\left(-\lambda t^{\alpha}\right)\right)^{\frac{1}{2}},
$$

This shows that the network system (1) is global MittagLeffler stable. This completes the proof.

Remark 3.1. It is easy to verify that $\eta>1$ in Theorem 3.3. Hence, from $\widetilde{\Delta}<0$, we can conclude that $\Delta<0$ holds. This shows that, under the assumption of Theorem 3.3, there exist a unique equilibrium point and a unique solution with the initial value for the network system (1).

Remark 3.2. In[13-19], Lyapunov function is constructed by using the absolute value function $V(t)=$ $\sum_{i=1}^{n} \mid x_{i}(t) \quad$. In [21-25, 35], Lyapunov function is considered by applying a positive definite quadratic function $V(t)=x^{T}(t) P x(t)$. However, in the proof of Theorem 3.3., Lur'e Postnikov type Lyapunov functional, which is composed of a positive definite quadratic function $\frac{1}{2} y^{T}(t) P y(t)$ and the integral term $\beta \sum_{i=1}^{n} \int_{0}^{z_{i}(t)} f_{i}(s) d s$, is utilized. It is easy to see that if $\beta=0$, Lur'e Postnikov type Lyapunov functional is changed into the common positive definite quadratic function. Moreover, in Lur'e Postnikov type Lyapunov functional, neuron activation function $f(x)$ is used, which makes the obtained results possess less conservatism.

In addition, compared with algebraic criteria in [1319], the stability condition established in terms of LMIs in Theorem 3.3 has a great application value in practical engineering.

\section{An illustrative example}

In this section, an example is provided to illustrate the validity with respect to the results in this paper.

Example 1. In the network system (1) with the piecewise constant argument $\gamma(t)$, we take the system parameters $\alpha=0.98$,

$A=\left(\begin{array}{cc}2 & 0 \\ 0 & 1.5\end{array}\right) . \quad B=\left(\begin{array}{cc}0.02 & -0.03 \\ 0.01 & -1\end{array}\right) . \quad C=\left(\begin{array}{ll}0.08 & 1 \\ 0.01 & 1\end{array}\right) . \quad I=\left(\begin{array}{l}1 \\ 1\end{array}\right)$. $\gamma(t)=\zeta_{k}, \zeta_{k}=\frac{2 k+1}{18}, k \in N . \theta_{k}=\frac{k}{9}$.

The active functions $f_{i}\left(x_{i}(t)\right)=\tanh \left(x_{i}(t)\right)+0.5 x_{i}(t)$, $i=1,2 . g_{1}\left(x_{1}(t)\right)=\tanh \left(\frac{x_{1}(t)}{7}\right)+0.5 x_{1}(t), g_{2}\left(x_{2}(t)\right)=$ $\tanh \left(\frac{x_{2}(t)}{6}\right)+0.5 x_{2}(t)$.

It is easy to verify that $L_{1}^{2}=L_{2}^{2}=3 / 2, L_{1}^{4}=9 / 14$, $L_{2}^{4}=2 / 3, m_{1}=2.0450, m_{2}=1.3333, \theta=1 / 9$, $E_{\alpha}\left(m_{1} \theta^{\alpha}\right)=1.2712, \eta=2.0321$, and

$$
\frac{\theta^{\alpha}\left(m_{1}+m_{2}\right)}{\Gamma(1+\alpha)}\left(\frac{1}{E_{\alpha}\left(m_{1} \theta^{\alpha}\right)}-\frac{m_{2} \theta^{\alpha}}{\Gamma(1+\alpha)}\right)^{-1}=0.6272<1,
$$

$\frac{m_{2} \theta^{\alpha}}{\Gamma(1+\alpha)}+\frac{m_{1} \theta^{\alpha}}{\Gamma(1+\alpha)}\left(1+\frac{m_{2} \theta^{\alpha}}{\Gamma(1+\alpha)}\right) E_{\alpha}\left(m_{1} \theta^{\alpha}\right)=0.5709<1$.

Obviously, the assumption (H1)-(H5) hold.
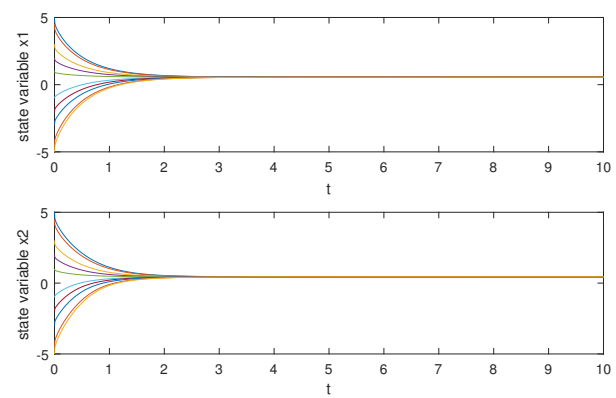

Figure 3: the state trajectory with different initial values of the network system (1) in Example 1

Set $\varepsilon=1$. In the following, we check the valid of Theorem 3.3. To do so, by using LMI toolbox for solving $\widetilde{\Delta}<0$ in Theorem 3.3, we get that

$$
P=\left(\begin{array}{cc}
0.0041 & 0 \\
0 & 0.5196
\end{array}\right)
$$

According to Theorem 3.3, we conclude that the unique equilibrium $x *=(0.5385,0.4424)$ of system (1) is Mittag-leffler stable, which is verified in Fig.3.

Remark 4.1. It is worthwhile pointing out that, due to the discontinuity of argument $\gamma(t)$, the solution of the network system (1) has the non-smoothness at the switching points $\theta_{k}, k \in N$, however, this is not seen in Fig.3. The reason is that we choose Lipschitz constants with respect to the activation functions and a sufficiently 
small $\theta$ to fulfill the assumptions of Theorem 3.3. So, the sufficient small $\theta$ "hides" the turning point.

By taking appropriate parameters of the network system (1), it enables that non-smoothness can be seen to implement. Consider the network system

$$
\begin{aligned}
{ }_{0}^{C} D_{t}^{\alpha} x(t)= & -\left(\begin{array}{cc}
20 & 0 \\
0 & 10
\end{array}\right)\left(\begin{array}{l}
x_{1}(t) \\
x_{2}(t)
\end{array}\right) \\
& +\left(\begin{array}{cc}
2 & 1 \\
8 & 0.2
\end{array}\right)\left(\begin{array}{l}
\tanh \left(x_{1}(t)\right)+0.1 x_{1}(t) \\
\tanh \left(x_{2}(t)\right)+0.1 x_{2}(t)
\end{array}\right) \\
& +\left(\begin{array}{cc}
1 & 20 \\
2 & 3
\end{array}\right)\left(\begin{array}{l}
\tanh \left(x_{1}(t)\right)+0.1 x_{1}(t) \\
\tanh \left(\frac{x_{2}(t)}{2}\right)+0.1 x_{2}(t)
\end{array}\right)
\end{aligned}
$$

where $\theta_{k}=\frac{k}{2}, \zeta_{k}=\frac{2 k+1}{4}, k \in N$. Obviously, in system (36), Lipschitz coefficient with respect to the activation function is bigger than the aforementioned in Example 1 , and $\theta=\frac{1}{2}>\frac{1}{9}$. The state of system (36) is depicted in Fig.4. From Fig.4, we can see clearly that the nonsmoothness of the solution has emerged at the switching point $\theta_{k}, k \in N$.

In addition, from Fig.4, we can see that, the solution of system (36) converges to the unique equilibrium $x^{*}=(0.7598,0.9944)^{T}$. However, it is easy to verify that the assumptions of Theorem 3.3 are not satisfied. This indicates that the assumption of Theorem 3.3 is only sufficient. That is to say, there exist some parameters, which do not satisfy the condition of Theorem 3.3, such that the network system (1) can also achieve the global Mittag-Leffler stability.
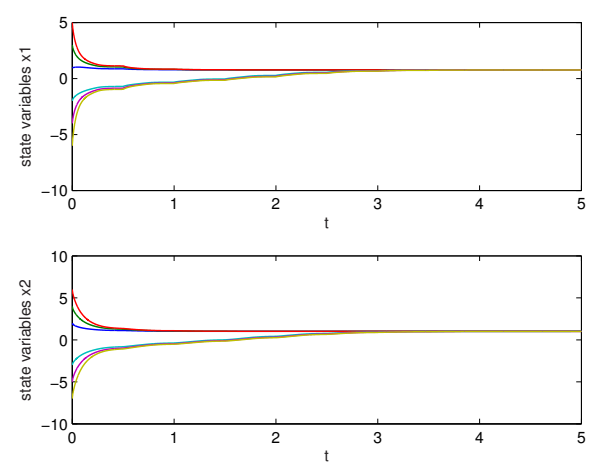

Figure 4: the state trajectory of the neural network system (36) with $\theta=1 / 2$

\section{Conclusion}

In this paper, the global Mittag-Leffler stability has be investigated for FNNs with piecewise constant argument. An inequality with respect to the fractional derivative of integer-order variable upper limit integral has been developed. The existence and uniqueness of equilibrium point and solution of FNNs with piecewise constant argument have been proved, respectively. Lur'e Postnikov type Lyapunov functional has be applied to address the Mittag-Leffler stability condition in terms of LMIs. Numerical example has shown the validity of the obtained results via Matlab toolbox.

It would be interesting to extend the results proposed in this paper to the synchronization of FNNs with piecewise constant argument. This issue will be the topic of our future research.

\section{Acknowledgment}

The authors are extremely grateful to anonymous reviewers for their careful reading of the manuscript and insightful comments, which help to enrich the content of the paper.

This work was jointly supported by the National Natural Science Foundation of China (61573306), the Postgraduate Innovation Project of Hebei province of China (00302-6370019), High level talent support project of Hebei province of China (C2015003054) and the LE STUDIUM RESEARCH PROFESSORSHIP award of Centre Val de Loire region in France.

\section{References}

[1] X. Xu, W. Tsai and N. Huang, Information capacity of McCulloch Pitts' model, Neural Networks, 1988(1): 149-158.

[2] J. Hopfield, Neural networks and physical systems with emergent collective computational abilities, Proc. Natl. Acad, 1982(79): 2554-2558.

[3] Y. Liu, S. Lee, O. Kwon, Ju H. Park. A study on $H_{\infty}$ state estimation of static neural networks with time-varying delays, Appl. Math. Comput., 2014(226): 589-597.

[4] S. Picozzi and B. West, Fractional Langevin model of memory in financial markets, Phys. Rev. E, 2002(66): 46-118.

[5] E. Reyes-Melo, J. Martinez-Vega, C. Guerrero-Salazar and U. Ortiz-Mendez, Application of fractional calculus to the modeling of dielectric relaxation phenomena in poly meric materials, J. Appl. Polym, 2005(98): 923-935.

[6] A. Kilbas, H. Srivastava and J. Trujillo, Theory and Application of Fractional Differential Equations, Elsevier, 2006.

[7] E. Ahmeda and A. Elgazzar, On fractional order differential equations model for nonlocal epidemics, Physica A, 2007(379): 607-614.

[8] G. Cottone, M. Paola and R. Santoro, A novel exact representation of stationary colored Gaussian processes (fractional differential approach), J. Phys. A, 2010(43): 84-85.

[9] N. Öalp and E. Demirci, A fractional order SEIR model with vertical transmission, Math. Comput. Modelling, 2011(54): 16.

[10] D. Liu, O. Gibaru, W. Perruquetti and T. Laleg-Kirati, Fractional order differentiation by integration and error analysis in noisy environment, IEEE T. Automat. Contr., 2015(60), 2945-2960. 
[11] X. Wei, D. Liu and D. Boutat, Non-Asymptotic Pseudo-State Estimation for a Class of Fractional Order Linear Systems, IEEE T. Automat. Contr., 2017(62), 1150-1164.

[12] H. Wu, X. Zhang, S. Xue and P. Niu, Quasi-uniform stability of Caputo-type fractional-order neural networks with mixed delay, Int. J. Mach. Learn Cyb., 2017(8): 1501-1511.

[13] J. Chen, Z. Zeng and P. Jiang, Global Mittag-Leffler stability and synchronization of memristor-based fractional-order neural networks, Neural Networks, 2014(51): 1-8.

[14] H. Wang, Y. Yu and G. Wen, Stability analysis of fractionalorder Hopfield neural networks with time delays, Neural Networks, 2014(55): 98-109.

[15] S. Zhang, Y. Yu and H. Wang, Mittag-Leffler stability of fractional-order Hopfield neural networks, Nonlinear Anal.: Hybri, 2015(16): 104-121.

[16] S. Zhang, Y. Yu and Q. Wang, Stability analysis of fractionalorder Hopfield neural networks with discontinuous activation functions, Neurocomputing, 2016(171): 1075-1084.

[17] R. Rakkiyappan, G. Velmurugan and J. Cao, Stability analysis of fractional-order complex-valued neural networks with time delays, Chaos Soliton Fract., 2015(78): 297-316.

[18] R. Rakkiyappan, G. Velmurugan and J. Cao, Analysis of global $o\left(t^{-\alpha}\right)$ stability and global asymptotical periodicity for a class of fractional-order complex-valued neural networks with time varying delays, Neural Networks, 2016(77): 51-69.

[19] A. Wu and Z. Zeng, Boundedness, Mittag-Leffler stability and asymptotical-periodicity of fractional-order fuzzy neural networks, Neural Networks, 2016(74): 73-84.

[20] H. Wu, X. Zhang, S. Xue, L. Wang and Y. Wang, LMI conditions to global Mittag-Leffler stability of fractional-order neura networks with impulses, Neurocomputing, 2016(193): 148-154.

[21] X. Yang, C. Li, Q. Song, T. Huang and X. Chen, Mittag-Leffler stability analysis on variable-time impulsive fractional-order neural networks, Neurocomputing, 2016, doi:10.1016/j.neucom.2016.04.045

[22] K. Cooke and J. Wiener, Retarded differentiaal equations with piecewise constant delays, J. Math. Anal. Appl., 1984(99): 265297.

[23] L. Dai, M.C. Singh, On oscillatory motion of spring-mass systems subjected to piecewise constant forces, J. Sound Vibration, 1994(173): 217-232.

[24] Y. Muroya, Persistence, contractivity and global stability in logistic equations with piecewise constant delays, J. Math. Anal. Appl., 2002(270): 602-635.

[25] X. Yang, Existence and exponential stability of almost periodic solution for cellular neural networks with piecewise constant argument, Acta Math. Appl. Sin., 2006(29): 789-800.

[26] H. Zhu and L. Huang, Dynamics of a class of nonlinear discretetime neural networks, Comput. Math. Appl., 2004(48): 85-94.

[27] P. Civalleri, M. Gilli and L. Pandolfi, On stability of cellular neural networks with delay, IEEE Trans. Circuits Syst. I, 1993(40): 157-164.

[28] M. Akhmet, On the integral manifolds of the differential equations with piecewise constant argument of generalized type, Proceedings of the conference on differential and difference equations at the Florida Institute of Technology, 2006: 11-20.

[29] M. Akhmet, On the reduction principle for differential equations with piecewise constant argument of generalized type, J. Math Anal. App., 2007(336): 646-663.

[30] M. Akhmeta, D. Arugaslan and E. Yilmaz, Stability analysis of recurrent neural networks with piecewise constant argument of generalized type, Neural Networks., 2010(23): 805-811.

[31] M. Akhmeta, D. Arugaslan and E. Yilmaz, Stability in cellular neural networks with a piecewise constant argument, J. Comput. Appl. Math., 2010(233): 2365-2373.
[32] T. Yu, D. Cao, S. Liu and H. Chen, Stability analysis of neural networks with peridic coefficients and piecewise constant arguments, J. Franklin I., 2016(353): 409-425.

[33] G. Bao, S. Wen and Z. Zeng, Robust stability analysis of interval fuzzy Cohen-Grossberg neural networks with piecewise constant argument of generalized type, Neural Networks, 2012(33): 32-41.

[34] W. Shen, Z. Zeng and S. Wen, Synchronization of complex dynamical network with piecewise constant argument of generalized type, Neurocomputing, 2016(173): 671-675.

[35] A. Wu, L. Liu, T. Huang and Z. Zeng, Mittag-Leffler stability of fractional-order neural networks in the presence of generalized piecewise constant arguments, Neural Networks, 2017(85): 118-127.

[36] I. Podlubny, Fractional differential equations. San Diego, California: Academic Press, 1999.

[37] L. Yan, Y. Chen and I. Podlubny, Stability of fractionalorder nonlianear dynamic systems: Lyapunov direct method and generalized Mittag-Leffler stability, Comput. Math. Appl., 2010(59): 1810-1821.

[38] H. Wu, Global exponential stability of Hopfield neural networks with delays and inverse Lipschitz neuron activations, Nonlinear Anal-Real, 2009(10): 2297-2306.

[39] H. Ye, J. Gao and Y. Ding, A generalized Gronwall inequality and its application to a fractional differential equation, J. Math. Anal. Appl., 2007(328): 1075-1081. 